\title{
e-Phaïstos
}

e-Phaïstos

Revue d'histoire des techniques / Journal of the history

of technology

IX-2 | 2021

Quel objet pour quel musée?

\section{Histoire de radars}

Applications des radars aux domaines de la météorologie et de la volcanologie à l'Observatoire de Physique du Globe de Clermont-Ferrand History of Radars at the Observatoire de Physique du Globe de Clermont-

Ferrand: Applications in Meteorology and Volcanology

Nathalie Vidal, Franck Donnadieu et Joël Van Baelen

\section{(2) OpenEdition}

Journals

Édition électronique

URL : https://journals.openedition.org/ephaistos/9128

DOI : $10.4000 /$ ephaistos. 9128

ISSN : 2552-0741

Éditeur

IHMC - Institut d'histoire moderne et contemporaine (UMR 8066)

Référence électronique

Nathalie Vidal, Franck Donnadieu et Joël Van Baelen, "Histoire de radars », e-Phaïstos [En ligne], IX-2 I

2021, mis en ligne le 26 octobre 2021, consulté le 28 octobre 2021. URL : http://

journals.openedition.org/ephaistos/9128; DOI : https://doi.org/10.4000/ephaistos.9128

Ce document a été généré automatiquement le 28 octobre 2021.

Tous droits réservés 


\section{Histoire de radars}

Applications des radars aux domaines de la météorologie et de la volcanologie à l'Observatoire de Physique du Globe de Clermont-Ferrand

History of Radars at the Observatoire de Physique du Globe de Clermont-

Ferrand: Applications in Meteorology and Volcanology

Nathalie Vidal, Franck Donnadieu et Joël Van Baelen

Remerciements

À Serge Godard, Roland Cordesses, Philippe Waldteufel, Jacques Kornprobst pour leurs informations précieuses relatives à l'histoire de l'Observatoire et des radars. À Bernard Delaunay pour sa relecture attentive et notre échange très riche au café des techniques.

\section{Apport de la technique des radars à l'étude et à la compréhension des phénomènes météorologiques}

\section{Les trois vies d'ANATOL (1970-1995)}

1 En 1948, Henri Dessens ${ }^{1}$ devient directeur de l'IOPG $^{2}$ et prend très vite conscience du besoin de radars à Clermont-Ferrand pour l'étude de la physique de l'atmosphère. Il obtint un financement pour l'acquisition d'un radar millimétrique ${ }^{3}$ mais le projet avorta $^{4}$. Henri Dessens, qui avait besoin d'un électronicien connaissant également la géophysique, fit appel à Serge Godard pour travailler sur son projet de radar météorologique ${ }^{5}$. Originaire de Gerzat dans le Puy-de-Dôme, il venait de terminer son cycle de licence à Paris, avec un certificat d'électronique ${ }^{6}$. Godard arrive à l'Observatoire début novembre 1959. Sous la direction de Dessens et du géophysicien Jean Coulomb ${ }^{7}$, il prépare une thèse de troisième cycle sur l'absorption des ondes électromagnétiques ultracourtes par les précipitations. Soutenue en 1966, elle met en évidence la possibilité d'utiliser un radar pour étudier la pluviométrie. 


\section{A l'origine, un radar militaire (1952-1968)}

2 Ayant réussi à trouver suffisamment de financements, Serge Godard recrute Roland Cordesses, un ingénieur, en 1968, en lui donnant pour premier travail de chercher un radar déjà existant ${ }^{8}$. Après plusieurs déplacements, un radar militaire est localisé sur la base de Francazal, près de Toulouse, mais dans un état médiocre et baignant dans l'eau'. Après deux jours d'analyse, il est décidé de le récupérer pour tenter de le remettre en fonctionnement, tout en l'adaptant à l'étude des météores. C'était un véritable pari.

3 Le radar appartenait à l'armée américaine. Fabriqué en 1952-1953, il avait servi durant la guerre de Corée ${ }^{10}$. Stocké en Afrique du Nord lors de la guerre d'Algérie ${ }^{11}$, il avait été finalement déposé à Toulouse, l'armée américaine ayant renoncé à son rapatriement du fait de son volume. C'est là qu'il fut repéré par Roland Cordesses. Pour le récupérer, Serge Godard échangea avec l'Inspecteur général de l'armée de l'Air, un contact d'autant plus fructueux que Godard avait suivi une École d'officiers, dans un service de recherche de l'armée de l'Air ${ }^{12}$. L'Inspecteur général, qui connaissait ses travaux sur les radars, lui proposa trois instruments remis à l'armée française par les Américains, faute de pouvoir les rapatrier. Serge Godard négocia leur récupération auprès du service de cartographie de l'armée américaine. La condition fut donc de récupérer les trois et de les rendre inutilisables sur le plan militaire. L'un arriva à Clermont-Ferrand, un autre fut installé à Lannemezan, station météorologique fondée par Henri Dessens ${ }^{13}$; le dernier, resté sur place à Francazal, fut utilisé comme source de pièces détachées à mesure des besoins afin d'entretenir celui de Clermont-Ferrand.

\section{Naissance d'ANATOL}

4 La démilitarisation fut effectuée en enlevant le calculateur analogique de tir muni d'un amplificateur opérationnel à tubes ${ }^{14}$. La poursuite automatique fut remplacée par une chaîne d'acquisition des données, pour laquelle Serge Godard s'était rapproché de l'entreprise Schlumberger et de son département «acquisition et traitement de données ».

5 Deux ans après sa récupération, sa réparation et sa démilitarisation, le radar météorologique était opérationnel à l'Observatoire. Baptisé ANATOL ${ }^{15}$ par Serge Godard, qui fut nommé à la direction de l'IOPG au décès de Dessens en 1971, il fut notamment utilisé pour faire de la trajectographie de cellules orageuses dans les Monts Dore ${ }^{16}$. L'équipe de l'Observatoire obtint ainsi des informations sur la forme et la nature des particules qui se trouvaient dans les nuages ${ }^{17}$. Il a également servi en 1973 pour des études d'orage dans la région d'olby, village situé dans la chaîne des Puys, près de Clermont-Ferrand.

6 Il fallait utiliser de grosses bandes dont la lecture n'était pas possible à l'Institut. Le journal local «La Montagne " possédait une machine adéquate. Un partenariat s'est noué entre les deux structures jusqu'à ce que le campus universitaire puisse acheter une machine pour assurer la lecture des bandes en question. Ce radar a donc tourné et a permis les débuts du traitement électronique du signal radar avec des échantillonneurs et des transistors spéciaux. 
Fig.1. Le radar ANATOL en action dans les Cévennes

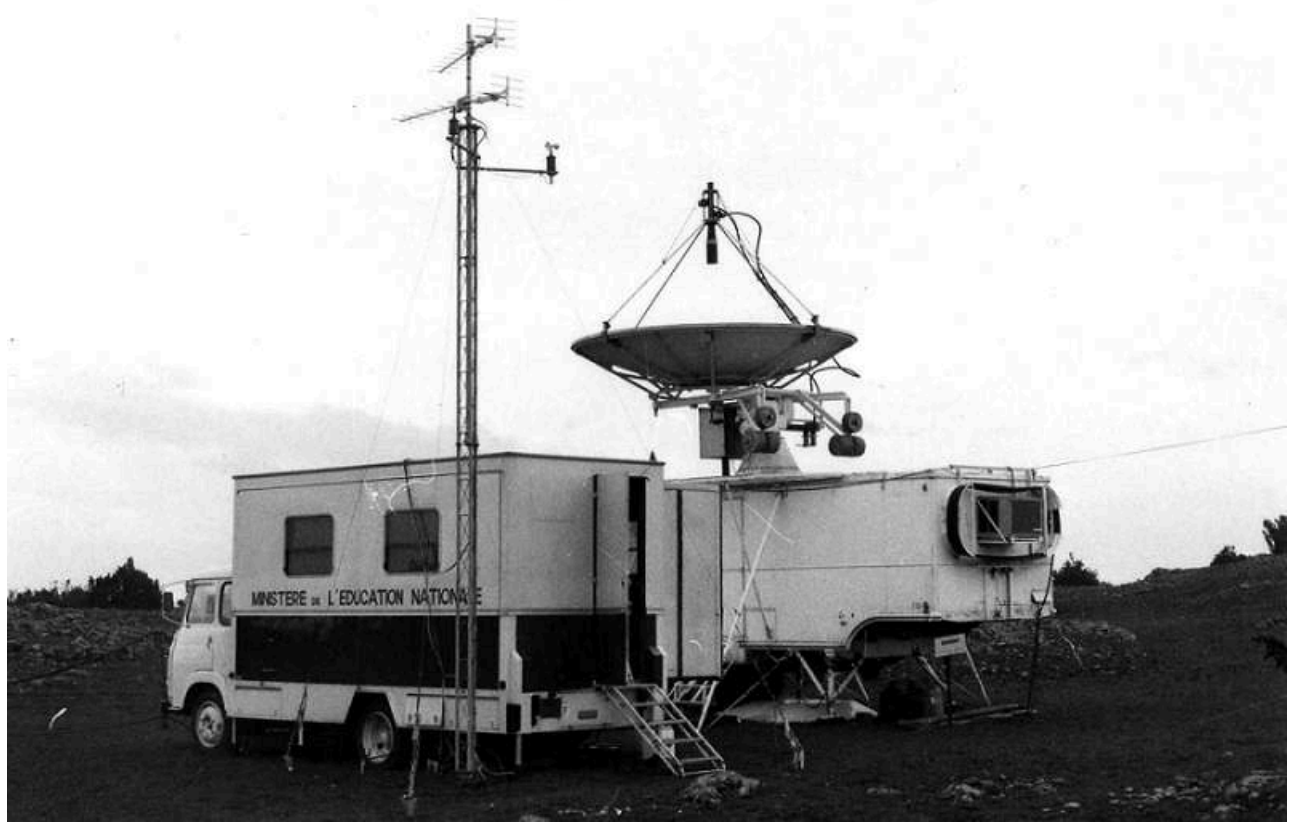

ANATOL (ANAlyse et Trajectoire d'Orages Locaux), radar militaire devenu radar météorologique à I'Observatoire de Physique du Globe de Clermont-Ferrand. Après de nombreuses campagnes et une carrière de 25 ans, ANATOL a été mis à la retraite mais sa tourelle a été conservée et installée sur le toit de l'OPGC.

OPGC

\section{Deuxième mission d'ANATOL}

Lors de sa prise de poste de directeur de l'Observatoire en 1977 et jusqu'en 1981, Philippe Waldteufel modifia ANATOL ${ }^{18}$. À partir de son travail réalisé dans le Colorado, il développa la radar-météorologie à l'Institut, avec une application à des campagnes de déclenchement de la foudre ${ }^{19}$, une campagne de surveillance d'orages à grêle en Suisse, une campagne en Côte-d'Ivoire, ainsi que le travail technique sur ANATOL et de nouvelles méthodologies applicables aux radars Doppler. Il s'est également intéressé au développement des radars acoustiques appelés sodars ${ }^{20}$. Ph. Waldteufel proposa aussi de développer des ballons d'altitude pour obtenir des données constantes; ce fut la phase "baba », qui a très bien fonctionné jusqu'à l'arrivée du GPS ${ }^{21}$. Ces ballons basse altitude étaient équipés de réflecteurs-radar ${ }^{22}$ permettant de restituer le champ de vent. En 1982, une étude de trajectographie de ballons à altitude constante pour la restitution du champ de vent fut lancée. Des ballons furent emportés par le vent et détectés par ANATOL pour restituer le champ de vent (région de Toulouse).

Philippe Waldteufel souhaitait pouvoir évaluer la quantité d'eau dans les nuages. Cette recherche se développa dans les années 80, toujours avec ANATOL. L'antenne fut conservée, mais les supports changèrent. Le principe consistait à envoyer des microondes dans l'atmosphère ${ }^{23}$. Philippe Waldteufel avait discuté avec Roland Cordesses de la faisabilité du projet. Ce dernier fut envoyé aux États-Unis se former à ce propos. Le projet fut ensuite repris par Daniel Ramond, qui dirigea l'Observatoire de 1982 à 1987. 


\section{5 ans de bons et loyaux services}

9 La troisième vie d'ANATOL, de nature hydrologique celle-là, fut également lancée par $\mathrm{Ph}$. Waldteufe ${ }^{24}$. En 1984 et 1985, une étude fut réalisée sur le bassin de l'Allier avec des pluviomètres installés au sol afin de comparer les données radar et les données au sol. Le système avait également été installé sur le plateau du campus universitaire des Cézeaux ${ }^{25}$. Une seconde étude météorologique fut menée en 1986, 1987 et 1988, au sud de Florac (Lozère), pour étudier les épisodes cévenols au pied du Mont Aigoual. Le radar avait été installé sur la commune de Barre des Cévennes, à $1030 \mathrm{~m}$ d'altitude ${ }^{26}$. Cette étude fut à l'origine de l'école d'hydrologie de Grenoble.

En 1992, ANATOL participa à une étude d'hydrologie urbaine à Saint-Denis (Roux 1996). En 1992 et 1993, c'est la commune urbaine de Bordeaux qui fit appel pour la dernière fois à ANATOL et ce jusqu'en 1995. Au total, ANATOL a donc été en activité pendant 45 ans.

11 Malgré son importance scientifique et ses 25 ans de carrière, ce radar n'a pas échappé à la destruction partielle. Installé sur un semi-remorque, il était très encombrant. Contacté, le directeur du Palais de la Découverte n'avait pu donner suite à une proposition de conservation. En 2005-2006, les domaines le vendirent comme radiotélescope à un groupe de radio amateurs de Corrèze... Il reste, comme unique trace, un fragment d'ANATOL (la tourelle) sur le toit de l'OPGC.

\section{Le radar ST (Stratosphérique-Troposphérique) ou radar profileur de vent VHF (Very High Frequency)}

Daniel Ramond lança avec Météo-France une opération qui consistait à organiser sur le territoire français un réseau de radars météorologiques pour la prévision à court et moyen terme : les radars ST (stratosphérique-Troposphérique). Il obtint des crédits pour en monter un à Clermont-Ferrand sur le terrain des Landais, là où était installé l'Observatoire à cette époque. Il s'agissait d'un radar de type Doppler déployé horizontalement. Il permettait de déterminer les gradients de vent en mesurant en continu les trois composantes du vent et en établissant le profil jusqu'à environ $15 \mathrm{~km}$ au-dessus du site. Mais l'opération menée par Météo-France fut orientée vers une autre thématique, ce qui eut pour effet de rendre orphelin le radar de Clermont.

Lorsque Jacques Kornprobst récupéra le projet en 1988, lors de sa prise de poste en tant que directeur de l'Observatoire, le cadre s'était complexifié, du fait des décentralisations administratives entreprises par le gouvernement ${ }^{27}$. Il avait décidé en effet d'implanter le Centre d'Étude du Machinisme Agricole et du Génie Rural des Eaux et Forêts, le CEMAGREF ${ }^{28}$, à Clermont-Ferrand, sur les terrains des Landais. Il fallut donc trouver de nouveaux locaux à la fois pour l'Observatoire et pour le radar ST. Une réunion avec le préfet posa les bases du nouveau bâtiment de l'OPGC en l'intégrant dans une enveloppe plus large, prévue pour la construction du pôle physique sur le campus universitaire des Cézeaux. Il fallut néanmoins attendre 1997 pour que l'OPGC rentre dans ses nouveaux bâtiments, soit à la fin du deuxième mandat de Jacques Kornprobst.

14 Mais le problème du radar ST restait pendant. Il ne pouvait pas être maintenu sur le site des Landais ni être installé sur le campus universitaire. Jacques Kornprobst reprit son bâton de pèlerin pour trouver un site adéquat afin d'assurer l'installation de ce 
radar. Une des premières solutions proposées fut le site de Marmilhat, dans la plaine de la Limagne. Mais, craignant des perturbations sur leurs propres radars, les autorités de l'aéroport d'Aulnat s'opposèrent au projet. Le contact pris avec la mairie de Romagnat, près de Clermont-Ferrand, s'avéra plus fructueux. Intéressé par le projet, le maire accorda un terrain à côté du cimetière, vers $0 \mathrm{pme}^{29}$. Le radar y fut réinstallé avec succès. En fonctionnement depuis 1998, il est toujours utilisé pour caractériser les gradients de vent au-dessus du puy de Dôme, pour travailler en liaison avec la station du puy de Dôme et le LAMP ${ }^{30}$.

\section{Les radars profileurs de pluie}

15 Depuis 2006, deux nouveaux types de radars vinrent s'ajouter aux réalisations antérieures de l'OPGC, Facilement transportables car peu volumineux, ils participèrent à des campagnes internationales, en complément des missions clermontoises ${ }^{31}$.

\section{Le radar en bande $X$}

16 Le radar en bande $\mathrm{X}$ est utilisé pour l'étude des précipitations météorologiques ${ }^{32}$. Réalisé en collaboration avec l'Institut Max-Planck et l'université d'Hambourg, le prototype de 2006 était au départ un radar de navigation commerciale. L'antenne d'origine fut remplacée par une antenne parabolique à faisceau fin et un système d'acquisition automatique ajouté. Le radar est installé sur une base ${ }^{33}$, sur le toit de l'OPGC, sur le campus universitaire des Cézeaux. Il mesure des valeurs de réflectivité qui servent à obtenir des images des nuages précipitants en PPI (Plan Position Indicator). Ce radar sert d'outil dans la prévision à courte échéance d'événements de pluies intenses et de risques d'inondation pouvant toucher une agglomération urbaine.

\section{Le Micro Rain Radar (MRR)}

17 Installé avec le radar ST au-dessus d'Opme, le Micro Rain Radar (MRR) de la société METEK est un radar Doppler ${ }^{34}$. Le traitement des données obtenues par le MRR permet de déduire la distribution spectrale des gouttes (en taille et en nombre) et les paramètres caractéristiques des précipitations (taux de précipitation, contenu en eau liquide...).

\section{Apport de la technique des radars à l'étude et à la compréhension des phénomènes volcanologiques}

\section{Naissance de VOLDORAD}

Entre 1993 à 1998, au cours de son second mandat comme directeur de l'observatoire, Jacques Kornprobst se consacra, entre autres, au développement d'un radar volcanologique. L'activité ANATOL, radar Doppler à diversité de polarisation, l'avait fortement intéressé, en effet. Il se pencha donc sur la question de l'effet Doppler et la technologie "profileur de vent» utilisée en météorologie. En 1993, il confia le développement de ce radar volcanologique à Roland Cordesses, devenu radariste de premier plan $^{35}$, et à l'équipe de l'Observatoire dont faisaient partie Georges Dubosclard, Jacques Fourné-Fayard, Claude Hervier et Yves Pointin, ce dernier pour le 
développement algorithmique initial (Dubosclard et al. 1999 et 2004). En parallèle, grâce au soutien de Claude Jaupart ${ }^{36}$, il obtient de la part de l'Institut National des Sciences de l'Univers (INSU), dans le cadre de l'ATP «aléas naturels " ${ }^{37}$, un budget suffisant pour réaliser l'opération qui consistait en un radar Doppler transportable sur les pentes des volcans.

19 Afin d'alléger l'appareillage, les antennes classiques furent abandonnées et le choix s'est porté sur une antenne Yagi, sorte de râteau de télévision extrêmement léger. La première expérience fut réalisée avec Patrick Allard, en 1998, sur les pentes de l'Etna ${ }^{38}$. Franck Donnadieu fut recruté pour développer ce radar baptisé VOLDORAD (Donnadieu et al. 2003 et 2005). Vulcanologue, enseignant-chercheur à l'observatoire, il est aujourd'hui en charge du service des radars Doppler volcanologiques de l'OPGC ${ }^{39}$.

\section{VOLDORAD, service international d'observation}

20 Il y eut en définitive quatre prototypes. VOLDORAD I, le plus volumineux, fut testé avec succès sur les pentes de l'Etna en 1998, en partenariat avec le CEA. Depuis fin mai 2013, il réalise le suivi de l'activité volcanologique du Popocatépetl au Mexique, en collaboration avec le CENAPRED ${ }^{40}$.

21 Ce succès à l'Etna conduisit rapidement à la construction de deux autres radars, VOLDORAD-2 et 2B. Plus compacts, ces radars sont uniques en volcanologie. VOLDORAD 2 fut le premier radar volcanologique vraiment transportable et opérationnel. Il fut notamment utilisé lors de trois paroxysmes de l'Etna en juillet 2001 puis au moment de l'éruption fissurale qui suivit et qui conduisit à la formation d'un nouveau cône nommé Laghetto. VOLDORAD-2B est intégré depuis 2009 au réseau de surveillance de l'Etna par l'Institut National de Géophysique et Volcanologie italien (INGV). Il a déjà enregistré une cinquantaine de paroxysmes à fontaine de lave engendrant des panaches de téphras jusqu'à $15 \mathrm{~km}$ d'altitude.

Fig.2. Les radars Doppler volcanologiques UHF (VOLDORAD) de l'OPGC
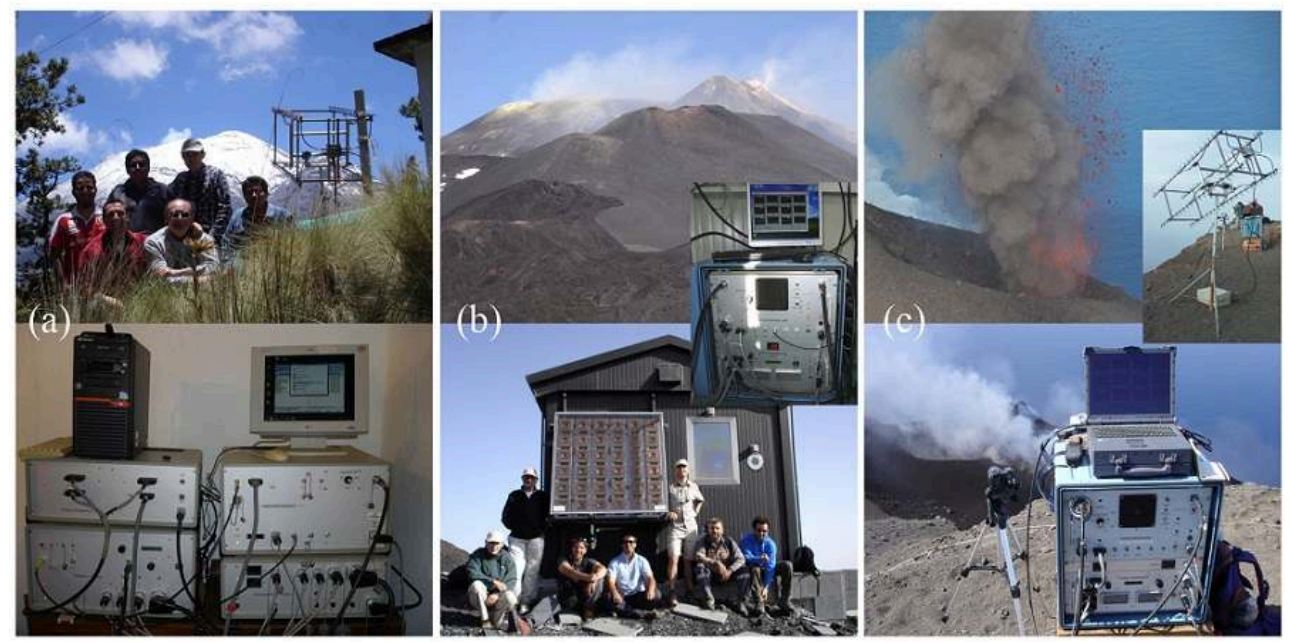

(a) Voldorad 1 effectuant le suivi continu du Popocatépetl (5426 m, Mexique) à $4000 \mathrm{~m}$ d'altitude ; (b) Voldorad 2B, avec son antenne micropatch, intégré au réseau de surveillance de l'INGV-CT à l'Etna ; (c) Voldorad 2, déployable en cas de crise ou pour des campagnes de mesures intégrées, ici à Stromboli en 2012.

OGCP 
Depuis, les VOLDORAD poursuivent leur activité internationale. Plusieurs campagnes de mesures ont été réalisées lors des éruptions de l'Etna, mais également sur différents volcans actifs dans le monde. Sur l'Arenal, volcan du Costa Rica, trois campagnes ont été organisées en 2004, 2005, et 2009, afin d'étudier la variabilité de la dynamique des panaches de cendres, D'autres études sont suivies, sur le Popocatépetl en 2007, le Yasur (Vanuatu) en 2008 et le Stromboli (Italie) en 2012 puis en 2015 (Valade et Donnadieu 2011, Donnadieu et Al. 2012, Freret-Lorgeril et al. 2018).

Le premier objectif était de définir les vitesses d'éjection du jet en mesurant la vitesse des particules par effet Doppler. Avec les calculs angulaires, il est possible en effet de déterminer une vitesse initiale du jet. Afin de répondre à ce premier objectif, deux jours d'expériences sur l'Etna ont été nécessaires pour recueillir plus de 1000 données contre trois autrefois par balistique à l'aide de caméras. Le deuxième objectif était de viser la charge en particules solides par unité de volume dans le panache. Avec la vitesse d'éjection du jet et la charge en particules solides, on peut modéliser la part d'éjectats solides dans le panache volcanique (Gouhier 2008, Gouhier et Donnadieu 2008).

24 À la suite des 3 premiers radars volcaniques, un nouveau radar scannant, à ondes millimétriques et effet Doppler (VOLDORAD-3), a été développé en collaboration avec un laboratoire atmosphérique spécifiquement pour le suivi des cendres volcaniques. Après une campagne de tests à Stromboli en 2015, toutes ses capacités de sondage en trois dimensions ont pu être mises en œuvre pour étudier les panaches de cendres plus imposants du volcan Sabancaya (Pérou) en $2018^{41}$.

Fig.3. VOLDORAD en action

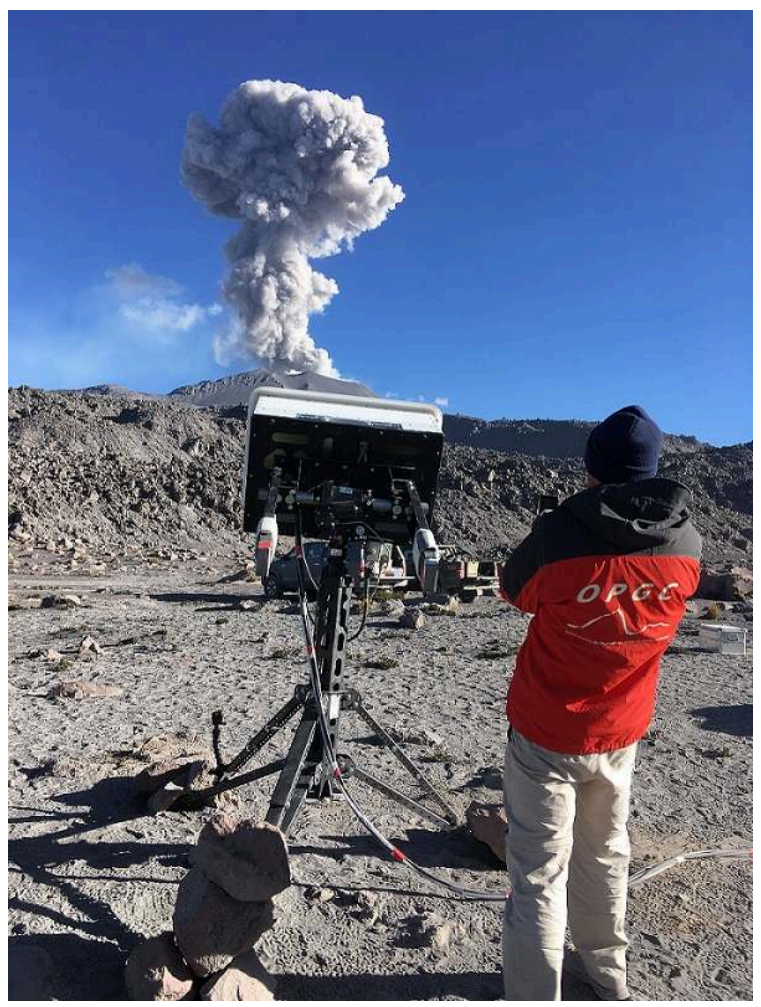

Le radar VOLDORAD-3 scanne à 4,5km de distance les panaches de cendres (ici environ $2 \mathrm{~km}$ de hauteur) du volcan Sabancaya (5967m) depuis une altitude de 5200m.

OGCP 
Réunis en un service d'observation dédié (VOLDORAD) depuis 2002 au sein de l'OPGC, ces quatre radars Doppler volcanologiques sont à l'origine de 12 campagnes de mesures sur 6 volcans actifs à travers le monde. Ils sont intégrés au sein du Service National des Observations en Volcanologie (SNOV). Les bases de données qu'ils permettent de réunir sont mises à disposition de la communauté scientifique.

\section{Conclusion}

Ces instruments scientifiques font l'objet d'un inventaire dans le cadre de la mission nationale de sauvegarde et de valorisation du patrimoine scientifique et technique contemporain (PATSTEC). L'inventaire est complété par l'interview d'anciens directeurs et ingénieurs de l'observatoire. Cette large collecte contribue à l'histoire d'une structure née en 1869 , qui remplit aujourd'hui à l'échelle internationale un rôle d'outil de recherche dans l'étude des phénomènes volcaniques et climatiques, en même temps qu'elle dresse l'histoire des instruments qui ont marqué sa recherche.

Depuis les prémices du projet d'observatoire météorologique, l'observatoire de Physique du Globe de Clermont-Ferrand a su se développer dans les domaines de la physique interne et externe en exploitant ses richesses locales que sont les volcans et notamment le puy de Dôme. Mais l'OPGC doit également son développement à son organisation originale, liant deux laboratoires : le laboratoire Magmas et Volcans (LMV) et le laboratoire de Météorologie Physique (LaMP), que l'on pourrait croire totalement opposés alors qu'ils sont complémentaires.

Un des liens forts entre ces deux départements réside notamment dans l'utilisation et le développement de radars. Le radar volcanologique VOLDORAD-3 en est un bon exemple : financé par des crédits interdisciplinaires du CNRS et du LabEx ClerVolc ${ }^{42}$, il est également utilisé pour des campagnes atmosphériques à l'instar de l'étude des orages et de la foudre en Corse (EXAEDRE en 2018), ou de la campagne bateau SEA2CLOUD du LAMP en 2019 destinée à mesurer les transferts d'aérosols entre l'océan et les nuages. Ce sont ainsi des équipes de recherche et des directeurs passionnés qui se sont succédé et se succèdent avec toujours la même volonté de faire avancer ce magnifique bateau qu'est l'OPGC.

\section{BIBLIOGRAPHIE}

ALLUARD Émile, De la fondation de l'Observatoire du puy de Dôme, Louis Bellet imprimeur-éditeur, Clermont-Ferrand, 1898 (a)

ALLUARD Émile, « De la fondation de l'Observatoire du puy de Dôme, notes et documents concernant l'histoire d'Auvergne », Bulletin histoire et scientifique de l'Auvergne, 1898 (b), p.99-107

ANDRIEU Hervé, CREUTIN Jean-Dominique, DELRIEU Guy, FOURNET Jacques, POINTIN Yves, «Hydrologie radar : bilan et perspectives », La Houille Blanche, n5-6, 1988, p.457-466 
DESSENS Henri, « L'Observatoire du puy de Dôme », L'enseignement scientifique supérieur, p.77-85

DONNADIEU Franck, DUBOSCLARD Georges, ALLARD Patrick, CORDESSES Roland, HERVIER Claude, KORNPROBST Jacques, LENAT Jean-François, « Sondages des jets volcaniques par radar Doppler : applications à l'Etna », Rapport quadriennal C.N.F.G.G. 1999-2002, 2003, p.119-124

DONNADIEU Franck, FREVILLE Patrick, HERVIER Claude, COLTELLI Mauro, SCOLLO S., PRESTIFILIPPO M., VALADE Sandrine, RIVET Sandrine, CACAULT Philippe, « Near-source Doppler Radar Monitoring of Tephra Plumes at Etna », Journal of Volcanology and Geothermal Research, vol. 312,2016 (a), p.26-39

DONNADIEU Franck, « Volcanological applications of Doppler radars: A Review and Examples from a Transportable Pulse Radar in L-band », in CHAU Jorge (eds.), InTechOpen, 2012, p.409-446

DONNADIEU Franck, FREVILLE Patrick, HERVIER Claude, LATCHIMY Thierry, PEYRIN Fréderic, RIVET Sandrine, CACAULT Philippe, GUEHENNEUX Yannick, « Des radars pour sonder l'intérieur des panaches volcaniques (VOLDORAD) », Revue d'Auvergne, Déc. 2016 (b), p.337-354

DONNADIEU Franck, DUBOSCLARD Georges, CORDESSES Roland, DRUITT Timothy, HERVIER Claude, KORNPROBST Jacques, LENAT Jean-François, ALLARD Patrick, COLTELLI Mauro, « Remotely Monitoring Volcanic Activity with Ground-based Doppler Radar », Eos, Transactions American Geophysical Union, vol.86, $\mathrm{n}^{\circ} 21,2005, \mathrm{p} .201-204$

DUBOSCALRD Georges, CORDESSES Roland, ALLARD Patrick, HERVIER Claude, COLTELLI Mauro, KORNPROBST Jacques, « First Testing of a Volcano Doppler Radar (Voldorad) at Mount Etna, Italy », Geophysical Research Letters, vol.26, n²2, 1999, p.3389-3392

DUBOSCALRD Georges, DONNADIEU Franck, ALLARD Patrick, CORDESSES Roland, HERVIER Claude, COLTELLI Mauro, PRIVITERA Eugenia, KORNPROBST Jacques, « Doppler Radar Sounding of Volcanic Eruption Dynamics at Mount Etna », Bulletin of Volcanology, vol.66, n5, 2004, p.443-456

FRERET-LORGERIL Valentin, DONNADIEU Franck, SCOLLO Simona, PROVOST Ariel, FREVILLE Patrick, GUEHENNEUX Yannick, HERVIER Claude, PRESTIFILIPPO Michele, COLTELLI Mauro, « Mass Eruption Rates of Tephra Plumes during the 2011-2015 Lava Fountain Paroxysms at Mt. Etna from Doppler Radar Retrievals », Frontiers in Earth Science, vol.6, 2018, p.73

GOUHIER Mathieu, Application du radar Doppler (VOLDORAD) à l'étude de la dynamique des éruptions stromboliennes de l'Etna, thèse de doctorat, Laboratoire Magmas et Volcans, Université Clermont Auvergne, 2008 (a)

GOUHIER Mathieu, DONNADIEU Franck, « Mass Estimations of Ejecta from Strombolian

Explosions by Inversion of Doppler-radar Measurements », Journal of Geophysical Research, vol.113, $\mathrm{n}^{\circ} \mathrm{B} 10,2008(\mathrm{~b})$

PLANCHE Céline, TRIDON Frédéric, MONIER Marie, VAN BAELEN Joël, « Les précipitations : observation et prévisions ", Des volcans aux Nuages, l'Observatoire de Physique du Globe de ClermontFerrand, Revue d'Auvergne, n620-621, 2016, p.99-124

POINTIN Yves, RAMOND Daniel, FOURNET-FAYARD Jacques, "Radar differential reflectivity Z DR: A real-case evaluation of errors induced by antenna characteristics", Journal of Atmospheric and Oceanic Technology, 1988, vol.5, $\mathrm{n}^{\circ} 3, \mathrm{p} .416-423$

VALADE Sandrine, DONNADIEU Franck, « Ballistics and Ash Plumes Discriminated by Doppler radar ", Geophysical Research Letters, vol.38, n²2, 2011

VIDAL Nathalie, « De l'Institut et Observatoire de Physique du Globe à l'Observatoire de Physique du Globe de Clermont-Ferrand ", Des volcans aux Nuages, l'Observatoire de Physique du Globe de Clermont-Ferrand, Revue d'Auvergne, $\mathrm{n}^{\circ}$ 620-621, 2016, p.39-56 
VIDAL Nathalie, «L'observatoire météorologique du puy de Dôme », Des volcans aux Nuages, l'Observatoire de Physique du Globe de Clermont-Ferrand, Revue d'Auvergne, 620-621, 2016, p.9-38

\section{NOTES}

1. L'idée d'un observatoire au sommet du puy de Dôme nait en 1869 avec Émile Alluard (1815-1908), professeur de physique à la faculté des sciences de Clermont-Ferrand. L'Observatoire météorologique du puy de Dôme, unique au monde, se consacre entièrement à l'étude des météores. Il se compose d'une station de plaine située en centre-ville et d'une station de montagne installée au sommet du puy de Dôme ; les deux entités étant reliées par le télégraphe. Émile Alluard fut le premier directeur de l'ensemble de 1879 à 1886. Henri Dessens (1911-1971), météorologiste, fut le sixième directeur de l'observatoire entre 1948 et 1969.

2. Le titre initial est Observatoire de météorologie du Puy de Dôme. Il devient IOPG en 1921, puis OPGC en 1985.

3. Ou radar à ondes millimétriques afin d'identifier les gouttelettes de pluie dont la taille ne dépasse pas 100 micromètres.

4. Il fut développé par le Laboratoire Central de Télécommunication (LCT) à Vélizy, dans les années 60 .

5. Serge Godard fut directeur de l'Observatoire de 1971 à 1976, puis maire de Clermont-Ferrand de 1997 à 2014, et sénateur du Puy-de-Dôme de 1998 à 2011.

6. Première année d'électronique à sa création

7. Normalien, agrégé de mathématiques, membre du groupe Bourbaki, Jean Coulomb (1904-1999) dirigea l'Institut de physique du globe de Paris de 1941 à 1959. Directeur général du CNRS de 1962 à 1967, il fut élu à l'Académie des Sciences en 1960. Il présida le CNES de 1962 à 1967, puis le Bureau des Longitudes, de 1967 à 1969.

8. Roland Cordesses fut recruté sur un contrat de recherche et de développement à durée déterminée, en partenariat avec l'armée, avant d'intégrer l'IOPG.

9. Radar militaire de type SCR-584 conçu en 1942 et mis au point en deux ans par le MIT (Massachussets Institut of Technology), aux États-Unis. Ce radar fut largement copié par tous les pays, car modulable et facile à mettre en œuvre.

10. De 1950 à 1953.

11. De 1954 à 1962.

12. Sur un sujet très original : le camouflage radar de bateaux de guerre avec des bulles de savon. Bien dimensionnées, les bulles de savon se comportent comme un véritable matelas.

13. Henri Dessens avait obtenu la création du centre de recherches atmosphériques (CRA) à Lannemezan, au pied des Pyrénées, en 1961, à Campistrous. Il y mit au point «une machine à faire pleuvoir", le "météotron». Jean Dessens, son fils, pionnier dans le recensement des tornades en France au XX ${ }^{\mathrm{e}}$ siècle, physicien à l'Observatoire Midi-Pyrénées, reprit les travaux de son père à son décès en 1971 ainsi que la direction du CRA. Il améliora le rendement du météotron en le rendant opérationnel en montagne, à une altitude de 2000 à 3000 mètres.

14. L'antenne militaire du radar fut changée pour en faire un radar millimétrique. L'antenne était très grande (4 mètres d'envergure) et très lourde ( 1 tonne). Il y avait en outre un important problème de prise au vent.

15. $A N A T O L=$ ANAlyse et Trajectoire d'Orages Locaux

16. Technique de télédétection active, un dispositif émet une onde grâce à une antenne et reçoit l'écho de cette onde rétro-diffusée renvoyée par le milieu sondé grâce à la même antenne. Le radar émettait pendant un temps très bref des ondes polarisées verticales ou horizontales.

17. Les grosses gouttes qui tombent lors des précipitations ont leur section verticale qui s'aplatit d'autant plus que leur diamètre est grand. Elles ont donc des sections de rétro-diffusion plus 
faibles en polarisation verticale qu'en polarisation horizontale. De plus, les gouttes d'eau de précipitation ne changent pas la polarisation de l'onde émise. Les mesures instantanées des réflectivités en polarisation horizontale et verticale sont donc en phase.

18. Polytechnicien spécialisé dans l'étude de l'ionosphère et de la thermosphère puis dans la radar-météorologie.

19. ANATOL fut utilisé sur le site de recherche sur la foudre, installé en Haute-Loire sur la commune de Saint-Privat-d'Allier. Initiée par le Commissariat à l'énergie atomique (CEA) et EDF, cette station a fonctionné pendant plus de 20 ans, de 1974 à 1996. Elle a accueilli des chercheurs du monde entier. Elle a également été à l'origine d'une première mondiale, avec la réalisation d'un éclair artificiel, en juillet 1973, déclenché sur un pylône situé sur le site et grâce au lancement de fusées depuis une aire de tir au sol. Inventaire PATSTEC Auvergne par Sébastien Lamy-au-Rousseau.

20. Sonic Detection And Ranging: c'est un appareil de télédétection en météorologie qui utilise les ondes sonores pour étudier les météores (vitesse et direction du vent entre autres) dans les basses couches de l'atmosphère terrestre.

21. Global Positioning System: système mondial de géo-positionnement par satellite (version américaine). Il existe aujourd'hui une version européenne (Galileo), une version russe (Glonass) et une version chinoise (Beidou).

22. Le réflecteur radar offre la plus grande et la plus réfléchissante surface possible pour faire écho à un signal radar.

23. Ou ondes hyperfréquences; si l'onde rencontre un obstacle, l'onde revient vers le radar. Le principe était d'émettre et d'écouter à partir d'impulsions d'une microseconde et pour une résolution de $100 \mathrm{~m}$. Les petites gouttes d'eau sont d'abord sphériques puis, quand elles grandissent, elles prennent une forme allongée. La réflectivité radar est plus ou moins importante selon la forme; le rapport des deux axes permet de déterminer la distribution des gouttes dans le nuage.

24. Ph. Waldteufel est également à l'origine du radar RONSARD toujours en activité et développé par le CNET (Centre National d'Études des Télécommunications, devenu France Télécom, puis Orange) de 1971 à 1974, radar moderne à effet Doppler. Ceci a également abouti à la création d'une école de radar-météorologie par Dessens. À l'époque, le développement des radars connaît une véritable révolution technologique.

25. Il s'agit au départ de la station de plaine créée par Alluard, implantée en ville dans une habitation louée (la tour de Rabanesse) dont l'état se détériore rapidement et ne permet plus d'assurer les mesures dans de bonnes conditions. Cette station de plaine aurait dû intégrer la faculté des sciences en tant que laboratoire mais les locaux étaient trop petits. La station de plaine déménage en 1913 sur la côte des Landais, près du plateau des Cézeaux, dans un bâtiment neuf construit spécialement pour l'Observatoire. L'OPGC est installé sur le campus universitaire depuis 1998.

26. Ce positionnement en altitude permet de limiter deux problèmes : le phénomène de blocage du faisceau qui induit une diminution du signal utile reçu et la détection d'échos de sol.

27. Devenu professeur de géologie à l'université de Clermont-Ferrand en 1973, Jacques Kornprobst créa le centre de recherches volcanologiques en 1984. Outre l'Observatoire, il dirigea le Laboratoire Magmas et Volcans de 1994 à 1996.

28. Centre d'Étude du Machinisme Agricole et du Génie Rural des Eaux et Forêts (CEMAGREF), devenu Institut national de recherche en sciences et technologies pour l'environnement et l'agriculture (IRSTEA), puis Institut national de recherche pour l'agriculture, l'alimentation et l'environnement (INRAE) en 2020.

29. Les premiers coups de pioche mirent au jour des objets archéologiques de l'âge du fer : le site se révéla être un avant-poste de Gergovie. Les archéologues intervinrent très rapidement. Heureusement, la mise en place du radar ST ne nécessita que quelques piquets à planter. 
L'installation est aujourd'hui complétée par des radars profileurs de vent installés sur le site d'Aulnat, dans la plaine de la Limagne.

30. Laboratoire d'atmosphère physique de l'Université Clermont Auvergne. L'OPGC est une entité de recherches qui regroupe également les laboratoires du LAMP et du LMV (Laboratoire Magmas et Volcans).

31. Convective and Orographically Induced Precipitation Study (COPS), Hydrological cycle in the Mediterranean experiment (HYMEX)

32. Radar météorologique de $3 \mathrm{~cm}$ de longueur d'onde et de $24 \mathrm{~kW}$ de puissance. Il fournit des données radiales de réflectivité tous les $90 \mathrm{~m}$ et jusqu'à $36 \mathrm{~km}$ de distance avec une résolution angulaire de $1^{\circ}$, toutes les 30 secondes. Il possède une haute résolution spatiale et temporelle : $60 \mathrm{~m}$ de distance, $1^{\circ}$ en azimut et $30 \mathrm{~s}$ en temps avec une portée effective de l'ordre de $20 \mathrm{~km}$ permettant de couvrir un petit bassin versant ou un bassin urbain.

33. Cette base est tout ce qui reste d'ANATOL.

34. En bande $\mathrm{K}(1,24 \mathrm{~cm}$ de longueur d'onde) à émission continue (FM-CW) de $50 \mathrm{~mW}$ de puissance fournissant le profil vertical des spectres Doppler tous les $100 \mathrm{~m}$ et jusqu'à $3 \mathrm{~km}$ de haut.

35. Roland Cordesses a obtenu le prix Cristal du Centre National de Recherche Scientifique (CNRS) pour l'ensemble de sa carrière et particulièrement pour la réalisation de ce radar volcanologique.

36. Volcanologue réputé, professeur de géophysique à l'université Paris-Diderot, membre de l'Académie des Sciences depuis 2018, Claude Jaupart a dirigé l'Institut de physique du globe de Paris, de 1999 à 2004, puis de 2011 à 2016.

37. Initiées en 1971, dans le cadre de la politique de recherche du CNRS, les Actions thématiques programmées (ATP), ont pour objectif de financer « des actions coordonnées, axées sur un thème déterminé, et portant sur la réalisation, en plusieurs années, d'un programme ", MARNOT Bruno, «Introduction au rapport d'activité du CNRS de 1969 », La revue pour l'histoire du CNRS, En ligne], $\mathrm{n}^{\circ} 10,2004$; DOI : https://doi.org/10.4000/histoire-cnrs.593

38. Chercheur au Centre des Faibles Radioactivités (CFR) de Gif-sur-Yvette à l'époque, devenu professeur émérite à l'Institut de Physique du Globe de Paris (IPGP), aujourd'hui président de la volcanologie internationale (IAVCEI).

39. Trois thèses ont également été soutenues sur le sujet, une autre débutant en co-tutelle avec le Department of Earth, Ocean and Atmospheric Sciences à Vancouver au Canada.

40. Centro National de Prevencion de Desastres (Mexique)

41. Les résultats prometteurs seront exploités dans le cadre d'une thèse en co-tutelle et d'un stage de recherche d'un étudiant en seconde année de Master.

42. Le label « laboratoires d'excellence» (LabEx) est décerné aux unités de recherche ayant une visibilité internationale, ainsi qu'une politique de recherche, de formation et de valorisation de haut niveau dans une thématique scientifique donné. «Labex» depuis 2011, ClerVolc (Centre Clermontois de Recherche sur le Volcanisme) associe 5 laboratoires de recherche de l'Université Clermont-Auvergne (UCA)-CNRS et de l'OPGC (Observatoire Physique du Globe de ClermontFerrand), sous le leadership du Laboratoire Magmas et Volcans de l'UCA. Voir: https:// clervolc.uca.fr. 


\section{RÉSUMÉS}

En 1970, Henri Dessens, directeur de l'Observatoire de Clermont-Ferrand, souhaite utiliser des radars pour l'étude de la physique de l'atmosphère. L'idée se concrétise avec Serge Godard, qui prend la suite de Dessens à la tête de l'observatoire. Il réussit à récupérer un radar militaire qui va être détourné de sa fonction première pour devenir un instrument d'étude et de recherche baptisé ANATOL (ANAlyse et Trajectoire d'Orages Locaux). La longue carrière d'ANATOL s'achève en 1995 après plus de 45 ans de bons et loyaux services dans la recherche atmosphérique. Mais l'idée d'utiliser des radars poursuit son chemin et dès 1993, sous la direction de Daniel Ramon, c'est l'installation d'un radar ST (Stratosphère-Troposphère) menée avec Météo-France. Puis, au milieu des années 1990, Jacques Kornprobst, directeur, lance l'Observatoire sur la conception et la réalisation technique d'un prototype de radar "volcanologique" à effet Doppler, VOLDORAD-1. Répondant à des besoins en volcanologie, il fut testé de façon pionnière sur le site de l'Etna en 1998. Ce succès conduisit dans la foulée à la construction de deux autres radars uniques en volcanologie (VOLDORAD-2 et $2 \mathrm{~B}$ ) puis d'un troisième (VOLDORAD-3). Réunis en un service d'observation dédié au sein de l'OPGC depuis 2002, ces radars sont utilisés pour la surveillance des éruptions volcaniques mais également pour des études atmosphériques.

In 1970, Henri Dessens, director of the Clermont-Fd Observatory, wanted to use radar to study the physics of the atmosphere. The idea became a reality with Serge Godard, who took over from Dessens at the head of the Observatory. He succeeded in recovering a military radar that was diverted from its original function to become a study and research instrument called ANATOL (ANAlyse et Trajectoire d'Orages Locaux). ANATOL's long career came to an end in 1995 after more than 45 years of good and loyal service in atmospheric research. However, the idea of using radars continued to grow and in 1993, under the direction of Daniel Ramon, an ST (StratosphereTroposphere) radar was installed with Météo-France. Then, in the mid-1990s, Jacques Kornprobst, director, launched the Observatory on the design and technical production of a prototype "volcanological" Doppler radar, VOLDORAD-1. Responding to volcanological needs, it was tested in a pioneering way at Etna in 1998. This success led to the construction of two other unique volcanological radars (VOLDORAD-2 and 2B) and a third (VOLDORAD-3). Since 2002, these radars have been combined into a dedicated observation service within the OPGC and are used for monitoring volcanic eruptions but also for atmospheric studies.

\section{INDEX}

Mots-clés : histoire des techniques, histoire des sciences, radar, doppler, instrument scientifique, culture scientifique et technique, patrimoine scientifique

Keywords : history of technology, history of science, radar, doppler, scientific instrument, scientific heritage, scientific and technical culture

Thèmes : Un objet une technique

\section{AUTEURS}

\section{NATHALIE VIDAL}

Nathalie Vidal est docteur en sciences de la Terre de l'Université Clermont Auvergne et titulaire du master histoire des sciences et techniques de l'université de Nantes/Centre François-Viète. 
Depuis 2005, elle est responsable du département Histoire des sciences et techniques au muséum Henri-Lecoq (Clermont Auvergne Métropole) et s'occupe notamment de deux originaux de la machine à calculer inventée et mise au point par Blaise Pascal. Elle est également responsable de la mission PATSTEC Auvergne qui a pour but l'inventaire, la conservation et la valorisation du patrimoine scientifique et technique contemporain. Elle est aussi membre associée du laboratoire Philosophie et Rationalité de l'Université Clermont Auvergne et poursuit des recherches dans les domaines des machines à calculer mécaniques, des observatoires météorologiques de montagne et plus particulièrement l'Observatoire météorologique du puy de Dôme.

\section{FRANCK DONNADIEU}

Franck Donnadieu est enseignant-chercheur (Physicien adjoint) au laboratoire Magmas et Volcans de l'Observatoire de Physique du Globe de Clermont-Ferrand (OPGC), au sein de l'Université Clermont Auvergne. Après une thèse en volcanologie en 2000 et des recherches postdoctorales aux USA, il s'est spécialisé dans le domaine pionnier de la volcanologie radar à l'OPGC avant son recrutement en 2002. Depuis une vingtaine d'années, il y dirige le service des radars

Doppler volcanologiques (VOLDORAD) comprenant quatre radars Doppler dont un contribuant à la surveillance de l'Etna en temps réel, et des bases de données. Ses travaux se consacrent à l'étude des éruptions explosives par radars Doppler transportables au travers d'une douzaine de campagnes de mesures sur différents volcans. Il est auteur/coauteur d'une trentaine de publications internationales et a rédigé trois chapitres d'ouvrage consacrés à la volcanologie radar. Habilité à diriger des recherches depuis 2017, il a encadré les travaux de quatre doctorants, trois post-doctorants, et une trentaine d'étudiants en Master.

\section{JOËL VAN BAELEN}

Joël Van Baelen est directeur de recherche au CNRS. Il a rejoint le LaMP (Laboratoire de Météorologie Physique) en 2004 et en a été le directeur-adjoint de 2008 à 2013 puis le directeur de 2014 à 2019. Il est maintenant directeur du LACy (Laboratoire de l'Atmosphère et des Cyclones) à La Réunion. Ses domaines de recherche se portent sur la télédétection atmosphérique appliquée à l'étude de la dynamique atmosphérique et des systèmes précipitant. En particulier, Joël Van Baelen s'intéresse à la distribution spatio-temporelle des pluies, à leur interaction avec le relief et à leur évolution dans le cadre du changement climatique. 